\title{
On a System of Equations
}

\author{
By H. Sircar
}

(Received 21st March, 1947. Read 2nd May, 1947.)

1. I propose to prove the following theorem.

With $n>2$, the $(n+2)$ equations derived from the matrix

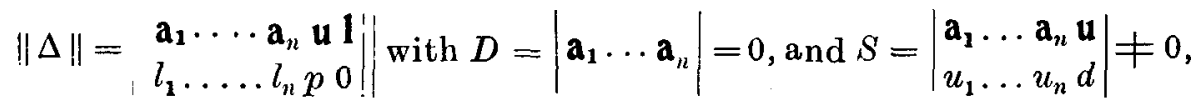
where

$$
\mathbf{u}=\left[\begin{array}{c}
u_{1} \\
\vdots \\
u_{n}
\end{array}\right], \mathbf{l}=\left[\begin{array}{c}
l_{1} \\
\vdots \\
l_{n}
\end{array}\right] \text { and } \mathbf{a}_{k}=\left[\begin{array}{c}
a_{\mathbf{1} k} \\
\vdots \\
a_{n k}
\end{array}\right], k=1,2, \ldots, n,
$$

by equating to zero all $(n+1)$-rowed determinants from the matrix $\|\Delta\|$ are equivalent to only two, one of which is linear in $l_{i}(i=1,2, \ldots, n)$ and the other is homogeneous and quadratic in a certain $n-1$ of $l_{i}(i=1,2, \ldots, n)$; the elements of the matrix are real; $a_{r 8}=a_{s_{r}}$ $(r, s=1,2, \ldots, n)$ and $d$ is arbitrary.

2. The following notations and symbols will be used. $\|M\|$ will mean a matrix ; $A_{i j}$ will denote the algebraic complement of $a_{i j}$ in $D$; $D^{\prime}$, the adjoint determinant of $D$; and

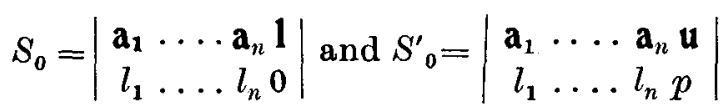

3. The following generalities may be noted.

3.1 The matrix of the adjoint determinant of a vanishing determinant has the rank one or zero.

3.2 In a symmetric determinant of rank $r$, there is a nonvanishing $r$-rowed principal minor.

$3.3^{1}$ If, in a matrix with $n+1$ rows and $n$ columns (or with

1 See Bôcher, Introduction to Higher Alygbru (1938), p. 58. 
$n$ rows and $n+1$ columns), two of the determinants of the matrix vanish, then the rank of the given matrix is $n-1$, provided that the matrix common to the two determinants is of rank $n-1$.

4. From the symmetry of $D$ follows that of $D^{\prime}$; and as $D=0$, $\left\|D^{\prime}\right\|$ has, according to 3.1 , the rank zero or one. $\left\|D^{\prime}\right\|$ cannot, however, have the rank zero, for, developing $S$ according to the theory of bordered determinants, we have

$$
D \neq S=-\sum_{i=1}^{n} \sum_{j=1}^{n} A_{i j} u_{i} u_{j},
$$

which would vanish, thereby giving rise to a contradiction, if $\left\|D^{\prime}\right\|$ had the rank zero.

$\left\|D^{\prime}\right\|$ must have, therefore, the rank one.

As $D^{\prime}$ is symmetric and $\left\|D^{\prime}\right\|$ has the rank one; one of its principal elements say $A_{i i}$, is, according to 3.2 , not equal to zero. We definitely choose $i$ for our future discussion.

From

$$
0 \neq S=-\sum_{i=1}^{n} \sum_{j=1}^{n} A_{i j} u_{i} u_{j}=-\left(\sum_{j=1}^{n} A_{i j} u_{j}\right)^{2} / A_{i i},
$$

in which we have made use of the equations

$$
A_{r q} A_{i i}=A_{i q} A_{r i}, \quad r, q=1,2, \ldots, n
$$

(which are derived from the consideration that $\left\|D^{\prime}\right\|$ has the rank one), follows

$$
\sum_{j=1}^{n} A_{i j} u_{j} \neq 0 .
$$

4.1 Consideration of the equation

$$
0=S_{0}=-\sum_{i=1}^{n} \sum_{j=1}^{n} A_{i j} l_{i} l_{j}=-\left(\sum_{=}^{n} A_{i j} l_{j}\right)^{2} / A_{i j},
$$

from the matrix $\|\Delta\|$, leads to the equation

We note that

$$
\sum_{j=1}^{n} A_{i j} l_{j}=0
$$

$$
D=0, \text { with } A_{i i} \neq 0 \text { and } \sum_{j=1}^{n} A_{i j} l_{j}=0 ;
$$

consequently the rank of the matrix

$$
\left\|\mathbf{a}_{1} \mathbf{a}_{2} \ldots \mathbf{a}_{n} \mathbf{1}\right\|
$$

is, according to $3.3, n-1$. 
Immediate consequences of (5) are that factorisation is possible, and that the coefficient of $p$ is zero, in the remaining equations from the matrix $\|\Delta\| ; p$ is, therefore, without influence and can be chosen arbitrarily.

4.2 The equation $S_{0}^{\prime}=0$ from the matrix $\|\Delta\|$ leads to

$$
\begin{aligned}
0=S^{\prime}{ }_{0} & =-\sum_{k=1}^{n} l_{k}\left(u_{1} A_{k 1}+u_{2} A_{k 2}+\ldots .+u_{n} A_{k n}\right) \\
& =-\left(\sum_{j=1}^{n} A_{i j} l_{j}\right)\left(\sum_{j=1}^{n} A_{i j} u_{j}\right) / A_{i i},
\end{aligned}
$$

in which we have made use of the relations

$$
A_{r q} A_{i i}=A_{i q} A_{r i}, \quad r, q=1,2, \ldots, n ;
$$

whence, in consequence of (3), we get the equation (4) (see 4.1); $S_{0}^{\prime}=0$ is, therefore; not an additional equation.

4.3 Since the quantities $A_{i 1}, A_{i 2}, \ldots, A_{i n}$ are not all zero $\left(A_{i i} \neq 0\right.$, see (2)), the equation

$$
\sum_{r=1}^{n} A_{i r} x_{r}=0
$$

has $n-1$ linearly independent solutions. Evidently, the vectors $\mathbf{a}_{k}(k=1,2, \ldots, n)$, of which exactly $n-1$ are linearly independent, form a complete, though redundant, set of solutions. On the other hand, we already have the equation (4) in 4.1, viz.,

Hence

$$
\sum_{r=1}^{n} A_{i r} l_{r}=0 \text {. }
$$

where the $\lambda$ 's are scalars.

$$
1=\sum_{i=1}^{n} \lambda_{k}^{-} \mathbf{a}_{k}
$$

Considering one of the remaining $n$ determinants, $f_{j}$ say, from the matrix $\| \Delta$ !, we have

$$
\begin{aligned}
& f_{j}=\left|\begin{array}{llllll}
\mathbf{a}_{1} \ldots & \mathbf{a}_{j-1} & \mathbf{a}_{j+1} \ldots & \mathbf{a}_{n} & \mathbf{u} & \mathbf{1} \\
l_{1} \ldots & l_{j-1} & l_{j+1} \ldots & l_{n} & p & 0
\end{array}\right| \\
& = \pm \lambda_{j}\left|\begin{array}{l}
\mathbf{a}_{1} \ldots \mathbf{a}_{j} \ldots \mathbf{a}_{n} \mathbf{u} \\
l_{1} \ldots l_{j} \ldots l_{n}
\end{array}\right| \pm Q\left|\mathbf{a}_{1} \ldots \mathbf{a}_{j-1} \mathbf{a}_{j+1} \mathbf{a}_{n} \mathbf{u}\right| \\
& \text { where } \\
& Q=\sum_{r=1}^{n} \lambda_{r} l_{r}
\end{aligned}
$$


The first determinant is $S^{\prime}$, which vanishes, and the second determinant, viz.,

$$
\left|a_{1} \ldots a_{j-1} a_{j+1} \cdots a_{n} u\right| \text {, }
$$

is different from zero when $j=i$ : see (3); and so from the equations

we must have $Q=0$.

$$
f_{1}=f_{2}=\ldots=f_{n}=0 \text {, }
$$

Eliminating from

$$
1=\sum_{r=1}^{n} \lambda_{r} \mathbf{a}_{r}
$$

one of the vectors, say $a_{i}$, where

$$
\mathbf{a}_{i}=\sum_{r=1}^{n} p_{r} \mathbf{a}_{r}
$$

with

$$
a_{k i}=\sum_{r=1}^{n} p_{r} a_{k r}, \quad k=1,2, \ldots, n,
$$

(the symbol $\sum_{r=1}^{n}$ denoting a summation in which $r$ assumes all values $1,2, \ldots, n$ excepting $i$; and the $p$ 's are scalars), we have

with

$$
1=\sum_{r=1}^{n}\left(\lambda_{r}+\lambda_{i} p_{r}\right) \mathbf{a}_{r}
$$

and

$$
l_{k}=\sum_{r=1}^{n}\left(\lambda_{r}+\lambda_{i} p_{r}\right) a_{r k}, \quad k=1,2 \ldots, i-1, i+1, \ldots, n,
$$

$$
l_{i}=\sum_{r=1}^{n}\left(\lambda_{r}+\lambda_{i} p_{r}\right) a_{r i}
$$

With the help of (8) and (9), it is easy to prove that

$$
l_{i}=\sum_{r=1}^{n} p_{r} l_{r}
$$

In consequence of $(10)$, the equation $\left(7^{\prime}\right)$ is reduced to

$$
\sum_{r=1}^{n}\left(\lambda_{r}+\lambda_{i} p_{r}\right) l_{r}=0
$$

Eliminating the $n-1$ constants $\lambda_{r}+\lambda_{i} p_{r}(r=1,2, \ldots, i-1$, $i+1, \ldots, n)$ from the equations (9) and (11), we have finally

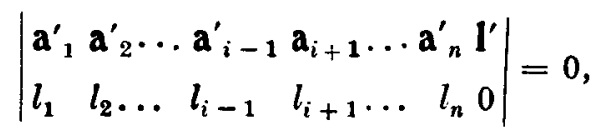


where

$$
\mathbf{1}^{\prime}=\left[\begin{array}{c}
l_{1} \\
\vdots \\
l_{i-1} \\
l_{i+1} \\
\vdots \\
l_{n}
\end{array}\right] \text { and } \mathbf{a}_{k}^{\prime}=\left[\begin{array}{c}
a_{1 k} \\
\vdots \\
a_{i-1, k} \\
a_{i+1, k} \\
\vdots \\
a_{n k}
\end{array}\right], k=1,2, \ldots, i-1, i+1, \ldots n .
$$

The equation (12) is homogeneous and quadratic in $l_{1}, \ldots, l_{i-1}, l_{i+1} \ldots, l_{n}$. We have hereby proved that the $n+2$ equations from the matrix $\|\Delta\|$ are equivalent to the equations (4) and (12).

I acknowledge my indebtedness to a referee, who has admirably simplified the proof of the theorem originally advanced by me. University OF DACCA,

BENGAL. 